\title{
Sistema de control basado en PLC y monitoreo mediante HMI para la automatización de una máquina de corte horizontal de bloques de esponja en el proceso industrial de fabricación de colchones
}

\author{
PLC-based control system and HMI monitoring for the automation of a sponge block horizontal \\ cutting machine in the industrial process of mattress manufacturing \\ E. Flores-García ${ }^{a}$, J.C. Quezada-Quezada ${ }^{b}$, L. Vargas-Téllez ${ }^{c}$, R.V.H. Calderón Medina ${ }^{d}$
}

\begin{abstract}
:
Nowadays, the industrial processes automation is an important part of development, productivity and optimization of processes or plants, providing quality, safety, speed, accuracy and economic benefit in manufacturing products or goods made through these processes. Automation can be found in a whole process, or in a stage of it, as the product requires. [1] [2]

Due to the inaccuracy and excessive waste of raw material which is presented as a problematic in the sponge mattresses manufacturing process in an industry in Tizayuca region, in this work a control system is proposed for the automation of a horizontal cutting machine for cutting sponge blocks of different sizes, and monitoring by a human-machine interface. [3] [4] [5]

For the proposed system conformation, it is considered the inclusion of convenient mechanical, electric and control elements, that can be easily acquired, and fulfill the required function for displacement and positioning of both, cutting knife and sponge, by using encoders, spindles and motors controlled by means of a PLC. [6] [7]

The proposed system represents a significant impact on the sponge cutting stage, since this contribute to improve the accuracy, quality and considerable reduction of raw material waste; it could be operated by a single operator, and the execution time at this stage will also be considerably reduced, consequently allowing a higher production. [8] [9]
\end{abstract}

Keywords:

Automation, encoder, HMI, Industrial process, PLC

Resumen:

Hoy en día, la automatización de procesos industriales forma parte importante en el desarrollo, productividad y optimización de los procesos o plantas, proveyendo calidad, seguridad, rapidez, exactitud y beneficio económico en la fabricación de productos o bienes que se elaboran en los procesos. La automatización puede ser encontrada en todo un proceso completo, o bien en una etapa del mismo, según el producto lo requiera. [1] [2] [10]

Debido a la inexactitud y el excesivo desperdicio de materia prima que se presentan como problemática en el proceso de fabricación de colchones de esponja en una industria de la región de Tizayuca, en el presente trabajo se propone un sistema de control para la automatización de una máquina cortadora horizontal para el corte de bloques de esponja de diferentes medidas, monitoreada a través de una interfaz humano-máquina. [3] [4] [5]

Para la conformación del sistema propuesto, se considera la inclusión de elementos mecánicos, eléctricos y de control convenientes, de fácil adquisición, que cumplen con la función requerida para el desplazamiento y posicionamiento tanto de una navaja de corte como de la esponja, mediante el uso de encoders, husillos y motores controlados por medio de un PLC. [6] [7] [11] [12]

El sistema propuesto representa un impacto significativo en la etapa de corte de esponja, ya que contribuye a mejorar la exactitud, calidad y reducción considerable del desperdicio de materia prima; podrá ser manipulado por un solo operador, y el tiempo de ejecución en esta etapa también se reducirá considerablemente, permitiendo consecuentemente una mayor producción. [8] [9]

Palabras Clave:

Automatización, encoder, HMI, PLC, Proceso industrial

${ }^{a}$ Autor de correspondencia, Universidad Autónoma del Estado de Hidalgo, Escuela Superior de Tizayuca, https://orcid.org/0000-0002-46068217, email: efloresg@uaeh.edu.mx

b Universidad Autónoma del Estado de Hidalgo, https://orcid.org/0000-0003-3125-3708, email: jcarlos@uaeh.edu.mx

c Universidad Autónoma del Estado de Hidalgo, https://orcid.org/0000-0003-2829-5210, email: leovargastellez@ gmail.com 


\section{Introducción}

La automatización de procesos industriales día a día requiere de actualizaciones y de personal altamente calificado para poder planear, diseñar, desarrollar, construir y operar sistemas automáticos que faciliten la fabricación de productos y que permitan la operatividad eficiente y segura de los procesos productivos [13] [14].

Dado el desarrollo vertiginoso de la tecnología, hoy en día se implementan sistemas automáticos diversos, sofisticados y complejos que facilitan la comunicación entre la máquina y el operador, permitiendo una mayor facilidad para la operación del sistema [15] [16].

Por lo anterior, en el presente trabajo se propone la implementación de un sistema de control adecuado a las características y los requerimientos del proceso de corte de bloques de esponja para la fabricación de colchones, mediante el desarrollo de un algoritmo de control a través del software Proficy Machine Edition de General Electric (GE), en lenguaje de programación Diagrama Escalera (LD, Ladder Diagram) [17], para efectuar el control retroalimentado de dos motores que, mediante acoplamientos mecánicos, actuarán el giro de una serie de husillos y éstos a su vez el desplazamiento lineal de una sierra y una plancha con la esponja, y con ello realizar el corte horizontal de los bloques de esponja [18]

Para el monitoreo del sistema, se propone el desarrollo de una Interfaz Humano-Máquina (HMI, Human-Machine Interface), que permita manipular tanto de modo manual como automático el proceso, permitiendo una sencilla operatividad y promoviendo la seguridad del operador y del sistema [19].

En el desarrollo del proyecto se hace uso del Modelo OSI, del protocolo de comunicación ethernet, de codificadores rotacionales, de un PAC-System RX3i y el módulo de comunicación ETM001, entre otros, que se describen brevemente en las siguientes secciones.

\section{Modelo OSI}

El modelo de interconexiones de sistemas abiertos, conocido como modelo OSI, es el modelo de una red descriptiva propuesta por una organización llamada ISO (Organización Internacional para Estandarización).

Este modelo está formado por siete capas que definen cada una de las fases y trayectos que recorren los datos para pasar de un dispositivo a otro mediante una red de comunicación, ello denomina un marco de referencia para la definición de la arquitectu ra de intercomunicación de los datos.
Este estándar es constituido como un modelo el cual puede servir como referencia para la construcción y arquitectura mediante el uso de otros protocolos y arquitecturas [20].

EI modelo OSI se basa en la idea de descomponer el proceso complejo de la comunicación en diversos problemas de menor complejidad, y así descomponerlos en cada una de las siete etapas de forma que cada una dependa de todas las demás; esto implica que cada etapa realiza trabajo que servirá o será de suma importancia para la capa superior [13].

\section{Protocolo de comunicación Ethernet}

Ethernet es un estándar de redes de computadoras de área local con acceso al medio por contienda CSMA/CD ("Acceso Múltiple por Detección de Portadora con Detección de Colisiones"). El nombre proviene del concepto físico de ether. Ethernet define las características de cableado y señalización de nivel físicoy los formatos de tramas de datos del nivel de enlace de datos del modelo OSI.

La Ethernet se tomó como base para la redacción del estándar internacional IEEE 802.3; usualmente se toman Ethernet e IEEE 802.3 como sinónimos. Ambas se diferencian en uno de los campos de la trama de datos; las tramas Ethernet e IEEE 802.3 pueden existir en la misma red [20].

\section{Señales de control}

Para el desarrollo y la automatización del sistema propuesto se utilizan dos tipos de señales, analógicas y digitales, las cuales pueden ser de entrada y/o de salida. Las señales analógicas son provenientes de una variación decimal, especificada dentro de un rango de valores asociados a señales de corriente 0 de voltaje, y corresponden, por ejemplo, a valores dentro del rango de 4 a $20 \mathrm{~mA}$, o de 0 a $10 \mathrm{~V}$ [21].

Las señales digitales son conocidas como todo o nada, ya que su valor de funcionamiento es 100 ; por lo tanto son binarias, e indican al operador, por ejemplo, el encendido o apagado de una lámpara, motor, etc. [22]

Ambos tipos de señales intervienen en este proyecto, puesto que se han utilizado señales de entrada digitales para botones de inicio o paro de la máquina, así como salidas digitales para alarmas visuales que indican el estado de la máquina, por ejemplo, si ésta está en funcionamiento o detenida. [6] 


\section{Lazo de control}

Para el control de la máquina, se relaciona un tipo de control on/off de lazo cerrado, que consiste en el encendido y apagado de motores a través de un PAC System RX3i de GE, un juego de contactores conectados a las salidas del PAC, y como lazo de retroalimentación un sensor de tipo encoder que, mediante una señal eléctrica, indicará la posición en la que se encuentre la sierra de la máquina, permitiendo detener o encender el motor en cualquiera de sus dos ejes de movimiento, según se requiera [23].

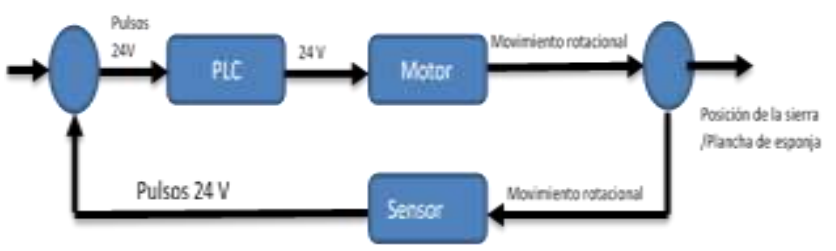

Figura 1. Lazo de control para la posición de la sierra y plancha de esponja

\section{Codificadores rotatorios (encoders)}

Los codificadores rotacionales, mejor conocidos como encoders, son sensores que pueden generar señales digitales en relación con el movimiento, que puede ser lineal o rotacional. Éstos pueden ser acoplados mecánicamente a diversos elementos en los que se requiera de una medición de precisión; por ejemplo, a engranes o flechas de motores, permitiendo la obtención de datos como posición del motor, velocidad, o en mecan ismos lineales para medir distancia; la obtención de datos se adquiere mediante señales digitales [7].

Hay dos tipos de éstos sensores, incrementales y absolutos; en general, el encoder de tipo incremental es aquel que genera pulsos mientras está en movimiento, ya sea lineal o rotacional; $y$ los absolutos, generan pulsos multi-bit que son codificados binariamente y permiten saber con mayor exactitud la posición del motor o elemento a controlar, además de que permiten la comunicación mediante redes industriales para mayor velocidad de respuesta y comunicación [7].

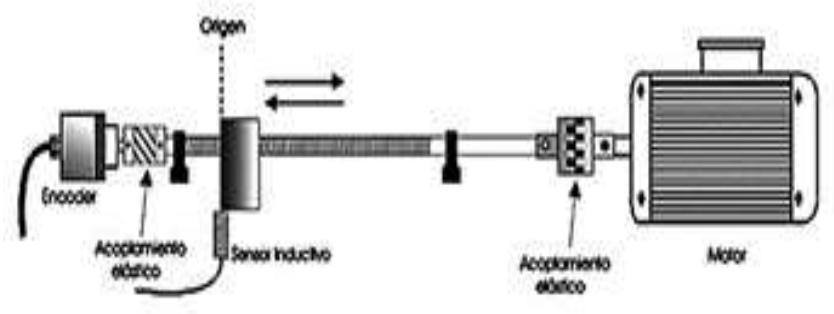

Figura 2. Acoplamiento de un encoder

\section{PAC System RX3i GE-Fanuc}

La implementación de un PAC System constituye el principal elemento de control para poder realizar la automatización de la máquina de corte, en un ambiente industrial, mediante el uso de señales digitales y analógicas [6] que son interpretadas por la memoria del PAC mediante la configuración de módulos de entrada, ya sea analógicos y/o digitales, y la construcción de un algoritmo en alguno de los distintos lenguajes de programación normados por la IEC 1131-3 [17].

A través del algoritmo, el programador realiza la adquisición y manipulación de las señales provenientes de los sensores para el accionamiento de salidas digitales que encenderán o detendrán, abrirán o cerrarán, activarán o desactivarán algún actuador implícito en el sistema [6].

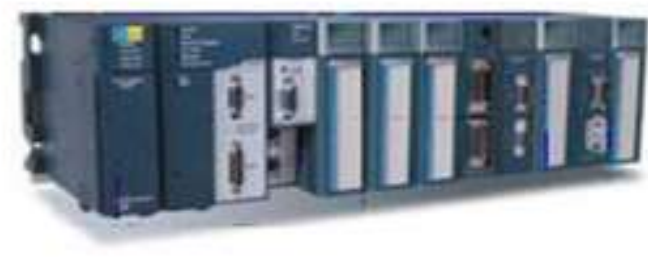

Figura 3. PAC System RX3i de GE

\section{Módulo de comunicación ETM001}

Este módulo permite realizar la comunicación mediante el protocolo Ethernetentre la computadora programadora, la interfaz humano-máquina, y el PAC.

El programador realiza la comunicación entre estos tres dispositivos por medio de la configuración y asignación de IP y mascara de subred.

El módulo ETM001 brinda flexibilidad para conectar de forma física el cable de datos mediante dos tipos de conectores; RJ45 y DB9 [6].

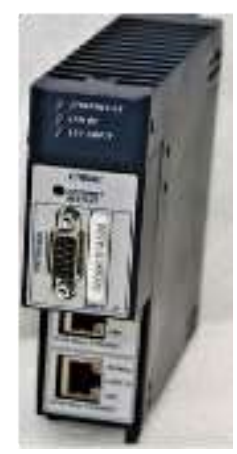

Figura 4. Módulo de comunicación Ethernet ETM001

Para la automatización del proceso de corte, se configura el módulo EMT001 mediante el software Proficy Machine 
Edition, la IP, y la máscara de subred, que corresponden a IP: 10.0.0.8, máscara de subred:255.255.255.0

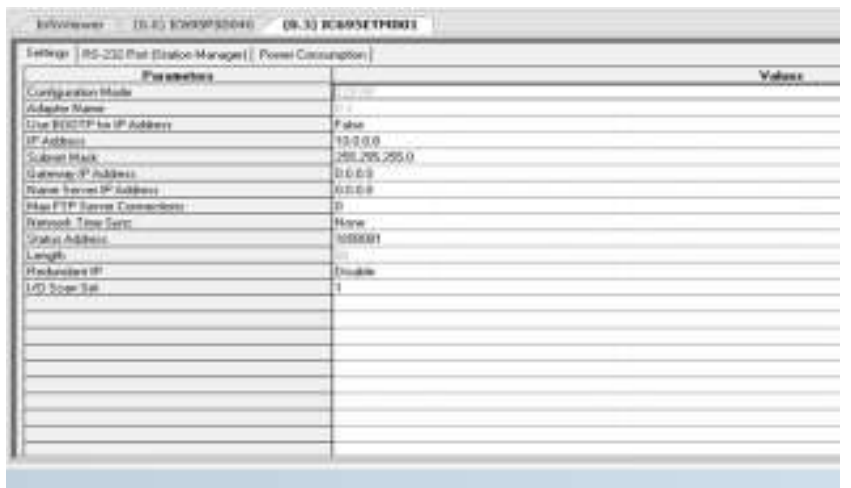

Figura 5. Configuración del módulo EMT001

\section{Módulo de salidas digitales IC694MDL754}

El módulo de salidas digitales proporciona 32 salidas divididas en dos grupos aislados de 16 cada uno. La conexión de estos dos grupos radica en la alimentación de cada uno en sus bornes positivos y negativos correspondientes. Estas salidas pueden conmutar voltajes de 12 a 24 VDC, con una corriente de 0.75 A por salida. Este módulo proporciona a cada salida un a protección de corto circuito, permitiendo trabajar a una temperatura de ambiente máxima de 60 grados centígrados.

En la Figura 6 se muestra un diagrama de conexión del módulo de salidas digitales; para la automatización del sistema se requieren cinco salidas digitales, las cuales, en este caso, son Q1, Q2, Q3, Q4 y Q5 [6], enmarcadas con un rectángulo en color rojo.
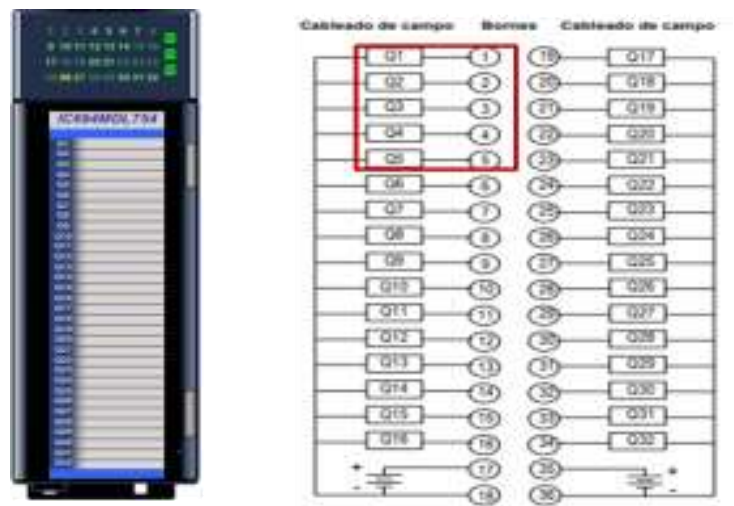

Figura 6. Módulo IC694MDL754 y diagrama de conexión

\section{Módulo de contadores rápidos IC695HSC308}

El módulo de contadores rápidos permite conectar, e incluir para la automatización del sistema, sensores tipo encoder, a valores de voltaje de 5, 12 y 24 VDC. Este módulo cuenta con 16 entradas de reloj y 14 salidas digitales, y tiene la capacidad de adquirir señales de pulso a una velocidad de $2.5 \mathrm{~ms}$

En la Figura 7 se presenta el diagrama de conexión del módulo de contadores rápidos, donde se pueden obsenar las entradas y salidas del módulo. Con base en este diagrama, se realiza la conexión de los sensores en las entradas Al1, Al2, Al3, y Al4, para la adquisición de las señales para detectar la posición de los motores a controlar. También se muestran, enmarcadas en rectángulos en color azul, las entradas utilizadas y la alimentación a $24 \mathrm{~V}$ del sensor [6].

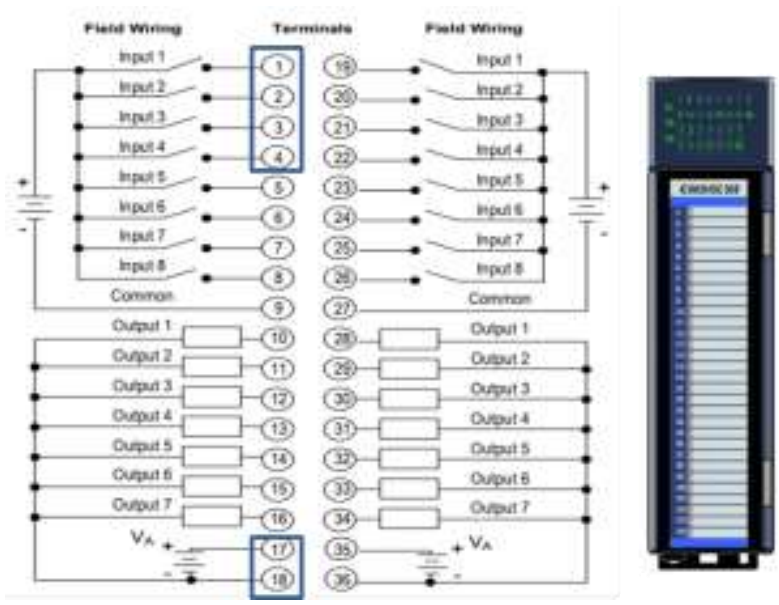

Figura 7. Diagrama de conexión y módulo IC695HSC308

\section{Configuración del contador rápido IC695HSC308}

Para poder realizar el control por retroalimentación mediante encoders, es necesaria la configuración del módulo mediante tres principales pasos:

1) Identificar y direccionar las entradas y salidas analógicas que se utilizaron en el algoritmo de control, que parten desde la entrada \%Al00001 y la salida digital $\%$ Q00001, y un nivel de voltaje de $24 \mathrm{~V}$.

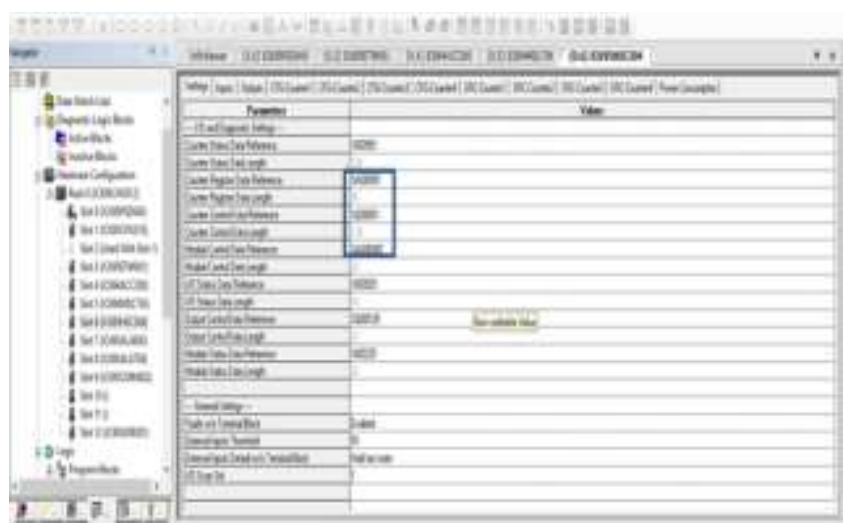

Figura 8. Configuración de entradas y salidas del módulo IC695HSC308 
2) Configuración del contador 1 y 2, identificando el tipo de encodera utilizar y la resolución del mismo.

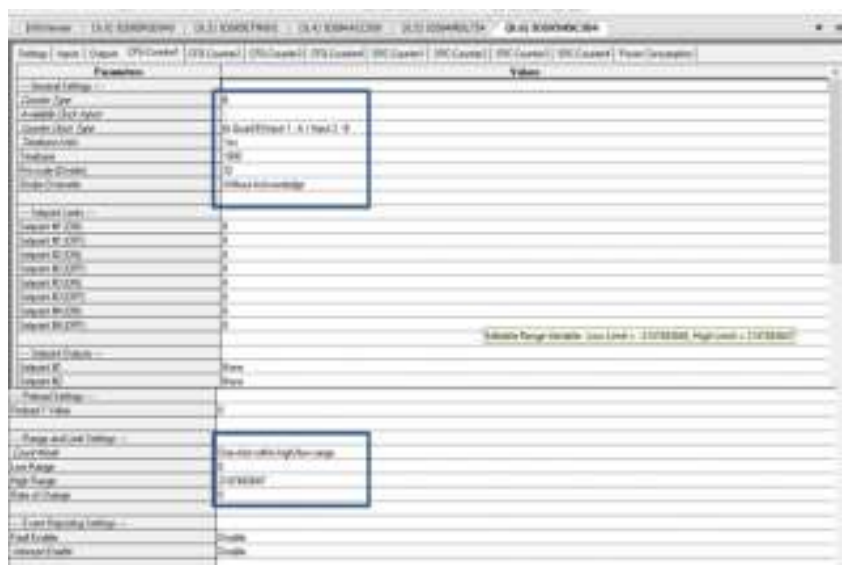

Figura 9. Configuración del contador para el tipo de encoder y su resolución

3) Configuración del reloj de entrada identificando las entradas que servirán para la adquisición de la señal analógica del sensor.

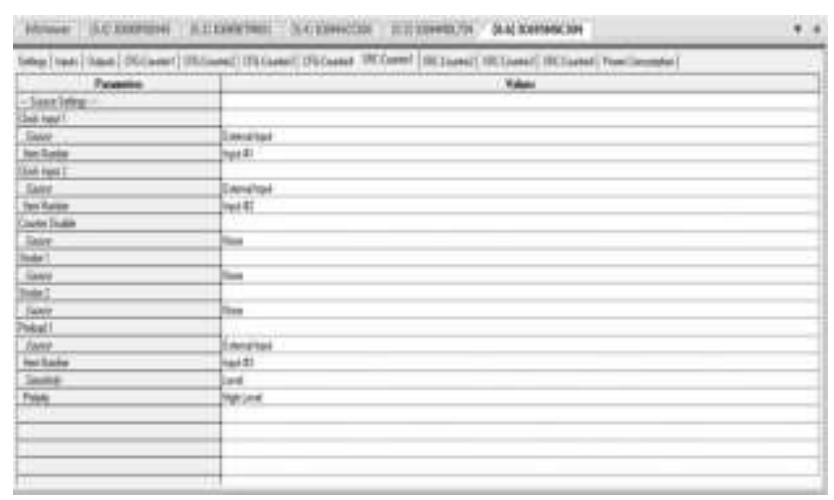

Figura 10. Configuración de las entradas de reloj

\section{Descripción del proceso}

En el presente trabajo, para la automatización del proceso industrial de corte en bloques de esponja para la fabricación de colchones, se implementa un PAC System $\mathrm{RX} 3 \mathrm{i}$, para la adquisición de señales de entrada analógicas y digitales, provenientes de los sensores tipo encodery fin de carrera [24] [25].

La implementación de la máquina de corte automatizada repercute en la sustitución del actual proceso realizado mediante una herramienta de corte de fabricación artesanal, elaborada por los mismos trabajadores. Esta herramienta de corte genera diversos desperfectos al realizar el corte, conllevando a que el producto (bloques de esponja) no cuente con la calidad necesaria, en comparación con otros dentro del mercado [26] [27]. Con base en este inconveniente, se desarrolla el diseño de un sistema de control capaz de atender las necesidades de este proceso; que brinde seguridad al operador y al sistema, y de fácil comprensión, manipulación y operatividad para el usuario. [28] [29] La fabricación de colchones de esponja engloba 4 etapas de proceso principales, a saber:

1) La primera etapa del proceso implementa la elaboración del bloque de esponja, la cual consiste en la mezcla de productos químicos que permiten la elaboración de la esponja; a esta etapa se le llama espumado [30].

Los productos químicos que intervienen son vaciados en un molde con medida de $2 \mathrm{~m}^{2}$, donde por reacción química se genera el bloque de esponja.

2) La segunda etapa, que es en la que está principalmente enfocado el sistema propuesto en este trabajo, es el corte de la esponja. Como se mencionó anteriormente, esta etapa se realiza actualmente mediante una herramienta de corte que inconvenientemente genera diversos desperfectos a la materia prima, impidiendo obtener la mayor calidad en el producto.

En esta etapa se realiza el corte de los bloques de esponja a medidas de corte especificadas por el cliente, o medidas estándar de colchones (altura de $5 \mathrm{~cm}, 10 \mathrm{~cm}, 18 \mathrm{~cm}$ y 22 $\mathrm{cm}$ ); el corte a tales medidas es realizado horizontalmente al bloque de esponja, para obtener planchas de esponja con altura determinada.

3) La siguiente etapa implementa el diseño y armado de las vestiduras o fundas del colchón; aquí, se diseñan las vestiduras de tela que cubrirán al colchón. Una vez finalizada la vestidura, se procede a forrar y coserla en la plancha de esponja.

4) Finalmente, la última etapa de elaboración consiste en envolvery sellar el colchón dentro de una bolsa de plástico con las dimensiones del colchón; a esta etapa se le denomina evidentemente sellado o embolsado.

\section{Algoritmo de control}

Para el control de corte de esponja se desarrolla un algoritmo de control programado en lenguaje LD mediante el software Proficy Machine Edition. Este algoritmo requiere el uso de una serie de bloques y contactos que permitan la fácil y correcta funcionalidad del sistema, mediante la adquisición de señales an alógicas y digitales, y con éstas ejercecer el accionamiento de manera manual/automática de los actuadores, es decir, los motores [17]. 


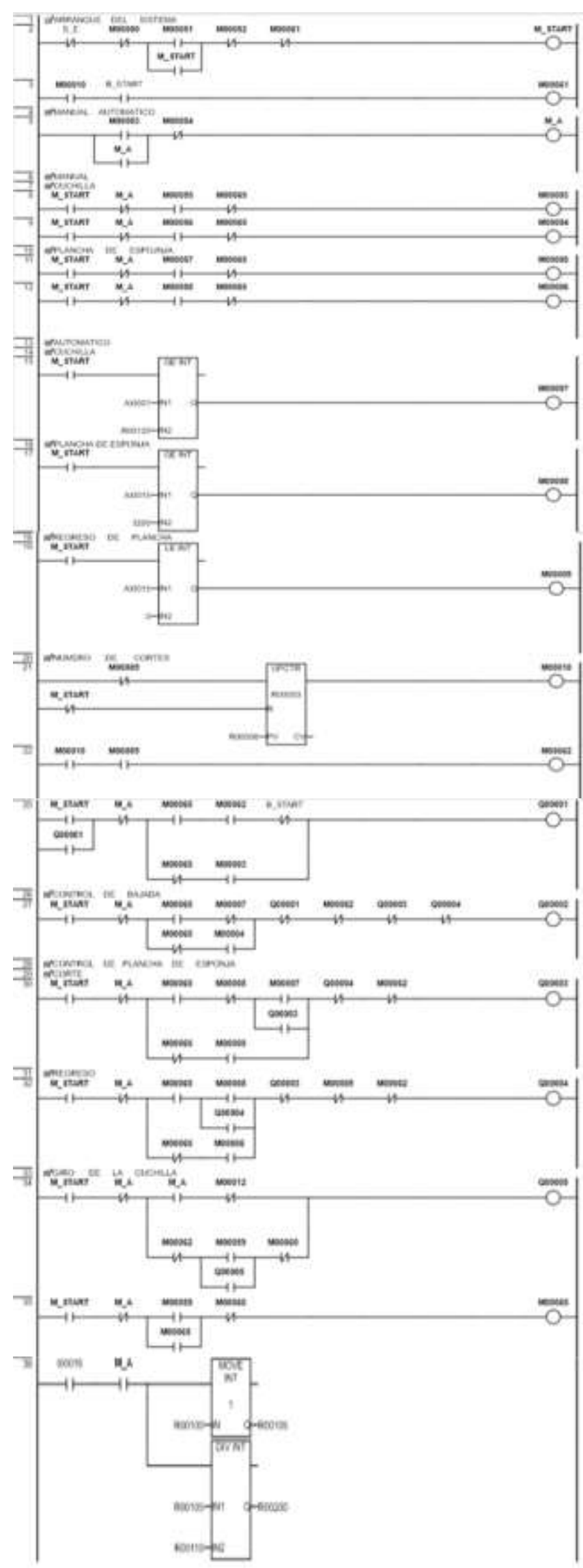

Figura 11. Líneas principales del algoritmo de control

\section{Tabla de direccionamiento}

Para comprender de mejor manera el algoritmo de control, se elabora una tabla de direccionamientos, la cual especifica el nombre, tipo y direccionamiento de cada contacto, y da una breve descripción [17].

Por medio de esta tabla, presentada como Tabla 1, se puede comprender, línea por línea, el funcionamiento del algoritmo, así como cada una de las operaciones de los contactos y bloques utilizados dentro del algoritmo.

\begin{tabular}{|c|c|c|c|}
\hline \multicolumn{4}{|c|}{ Tabia de direccise amientos } \\
\hline Variable & Tego & Directootamiento & Description \\
\hline AlDON1 & Analogica de entrida & AlWOOI & Entreda dal encoter 1 \\
\hline AlDo:15 & Analogica de entredta & AlDOO15 & Entrada del encoter? \\
\hline B.START & Discreta fisia de entreda & soomi & $\begin{array}{l}\text { Bocon fisico que pore en martha el } \\
\text { sistema }\end{array}$ \\
\hline |tom:6 & Discoeta fisia de entrada & 80016 & $\begin{array}{l}\text { Entreda fisica qua reinicia el bloqua } \\
\text { de chiobot en moto autsmation. }\end{array}$ \\
\hline Moccos & Memoria discoteta & $\operatorname{sincons}$ & Mamoria de nodo man.al \\
\hline swoos & Memoria discoeta & svocoos & Mamoria de nodo manaal \\
\hline vecos & Memoria discreta & sucous & |Memoria de nodo manual \\
\hline scecos & Memoria discreta & sutoos & Memoria de nodo manual \\
\hline secos & Memoria discreta & sucous & $\begin{array}{l}\text { Menoria que detendre al motse lett } \\
\text { mods automatico. }\end{array}$ \\
\hline мw0:8 & Memoria discreta & swomos & $\begin{array}{l}\text { Memoria que detendra al moter } 2 \text { en } \\
\text { modosatomatica. }\end{array}$ \\
\hline swoses & Memoria discreta & siveores & $\begin{array}{l}\text { Memoria que detiene la maquina } \\
\text { cuands semina de realizarlos cottes } \\
\text { en nodo man al y ausamatico }\end{array}$ \\
\hline newo10 & Memoria discreta & svcouto & $\begin{array}{l}\text { Memoria que detiene al motor } 2 \\
\text { cuando re ejega a su posiobn inicial }\end{array}$ \\
\hline $\operatorname{M\omega OSO}$ & Memoria discreta & svomso & Mamoria dinecrionada al HWI de STOP \\
\hline мwos! & Memoria discreta & sucoosi & Memoria divectionada al HVI de start \\
\hline Nowes & Memoria discreta & svo002 & Menoria divectionada al HWI de Soop \\
\hline $\operatorname{voco63}$ & Memoria discreta & sucots & Memoria de nodo manual \\
\hline МWOSA & Memaria discreta & sucosas & Memoria de nodo autam atico \\
\hline scouss & Memoria discreta & sucous & $\begin{array}{l}\text { Memoria de martha en modo maual } \\
\text { del motor } 1\end{array}$ \\
\hline scoos & Memoria discreta & $8 v 0006$ & Memoria de neøeso del notser 1 \\
\hline $\operatorname{secos} 7$ & Memoria discreta & svowo & $\begin{array}{l}\text { Memoria de martha en modo manual } \\
\text { del motor } 2\end{array}$ \\
\hline $\operatorname{Mecos8}$ & Memoria discreta & swowss & Mamoria de negreso del notsr 2 . \\
\hline $\operatorname{swc69}$ & Memoria discreta & sNowos & $\begin{array}{l}\text { Memoria que aciona lacuchilaten } \\
\text { modo manual. }\end{array}$ \\
\hline swowo & Memoria discreta & sucosid & $\begin{array}{l}\text { Memoria que detiene la cuchilla en } \\
\text { modo manual. }\end{array}$ \\
\hline мw061 & Memoria discreta & suconsil & Memoria que detiene toto el sistema \\
\hline Q00001 & Salida discreta & 500001 & Salisade start del motor 1 \\
\hline 0,0008 & Salida discreta & 900000 & Salitade refreso del motor 1 \\
\hline COONOS & Salidadiscreta & YOPOOS & Salisade stan sel motor? \\
\hline 0,0004 & Salida discreta & 500004 & Salisade regreso del motor 2 \\
\hline COODOS & Salida discreta & Yopooss & Salisa para accoonar la serra \\
\hline
\end{tabular}

Tabla 1. Direccionamientos en el algoritmo de control 


\section{Interfaz Humano Máquina (HMI)}

Para la interacción entre el operador y la máquina, se diseña una $\mathrm{HMl}$; esta interfaz permite al operador reprogramar las medidas a las que se deberán llevar a cabo los cortes. Además, permite que el usuario visualice de forma gráfica el movimiento en ambos ejes, tanto de la plancha de corte como de la sierra cortadora.

El operador puede seleccionar entre los modos de operación manual o automático, y realizar ajustes de posición de ambos componentes según las medidas del bloque lo requieran.

La Figura 12 muestra el diseño de la HMI, tal como el operador la visualizará en su panel de control; el modo en que se encuentra principalmente es automático, donde el operador indicará la medida del bloque de esponja y la medida de la altura de los cortes, con lo cual la interfaz automáticamente indicará la cantidad de cortes que se pueden realizar con esas características.

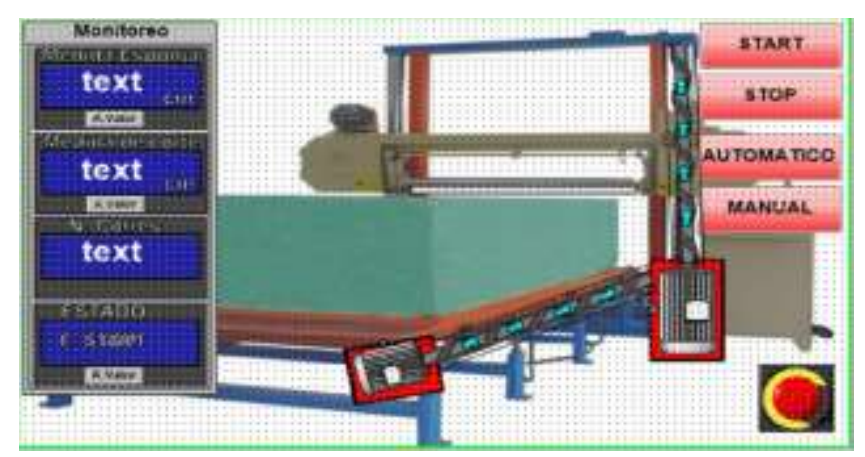

Figura 12. HMI en modo automático

La Figura 13 muestra el diseño de la HMI en modo de operación manual, tal como el operador la visualizará en su panel de control, con la que podrá ajustar la máquina en la posición que desee; una vez posicionada, indicará las medidas de corte y cuántos cortes requiere realizar. Especificados esos valores, al presionar el botón Inicio la interfaz causará que la máquina comience a operar con los valores asignados por el operador.

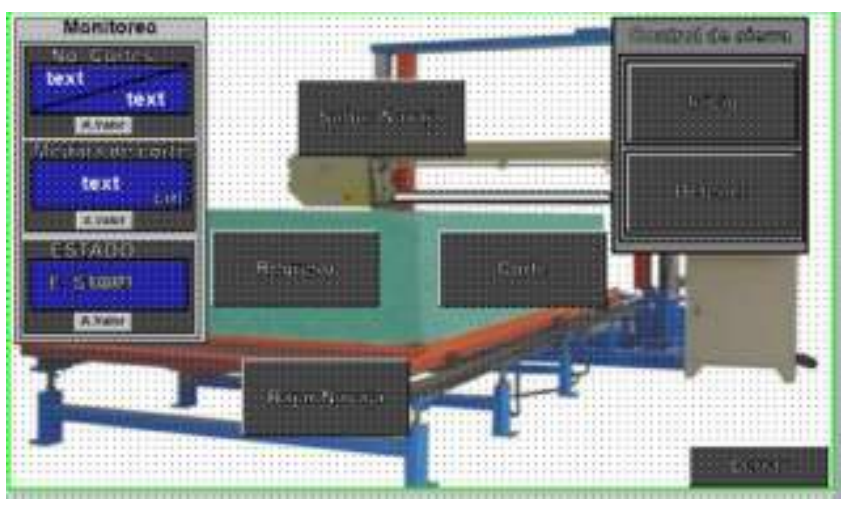

Figura 13. HMI en modo manual

\section{Conclusiones}

Hoy en día la automatización de procesos industriales juega un papel importante dentro de la actualización e innovación de sistemas de control, para brindar un mejor servicio o producto con mayor calidad al mercado.

Dada esa necesidad, en este trabajo se ha propuesto un sistema de control que ofrece seguridad y fácil operatividad al operador, mediante el diseño de una interfaz comunicada mediante protocolos que permiten incrementar la velocidad de respuesta del sistema.

El funcionamiento meramente mecánico constituye un factor determinante, debido a que los errores de los acoplamientos mecánicos pueden causar inconvenientes - lecturas erróneas por medio del encoder, lo que introduce un margen de error al sistema, solucionándolo mediante la inclusión de un sensor de mayor resolución y ajustando con mayor precisión el motor junto con el husillo y el encoder.

Finalmente, las pruebas realizadas y el ajuste de parámetros en el sistema dieron una serie de resultados satisfactorios con un algoritmo y una HMl funcionales, eficientes y operables de forma sencilla para el operador.

\section{Referencias}

[1] Butech, «Maquinaria de corte y esponja,» Herramientas de corte, vol. $6, \mathrm{n}^{\circ} 3$, p. 25, Septiembre 2017.

[2] E. Ganchozo Loor Winter y E. Guanga Cuadrado, «Diseño y construccion de un equipo de corte horizontal para bloques de espuma de poliuretano, en cepolfi industrial,» 18 Octubre 2017. [En línea]. Available: http://dspace.espoch.edu.ec/handle/123456789/895. [Último acceso: 15 Febrero 2019].

[3] J. Arroyave Londoño, «Ingeniería inversa de un reductor de tornillo sinfín,» 19 Junio 2013. [En línea]. Available: https://dialnet.unirioja .es/servlet/articulo?codigo=4271876. [Último acceso: 20 Febrero 2019].

[4] A. Checa Rodríguez, «CÁLCULO Y DISEÑO DE ELEVADOR MEDIANTE HUSILLO,» 23 Abril 2018. [En línea]. Available: https://idus.us.es/xmlui/handle/11441/75132. [Último acceso: 25 Febrero 2019].

[5] U. C. C. Herrera, «PRINCIPIO DE ARQUÍMEDES, ESPIRAL DE ARQUÍMEDES O TORNILLO DE ARQUÍMEDES,» 13 Mayo 2005. [En línea]. Available: https://blog.uchceu.es. [Último acceso: 20 Febrero 2019].

[6] C. E. Internacional, «Programmable controllers,» 5 Mayo 2003. [En línea]. Available: https://www.iec.ch/. [Último acceso: 28 Febrero 2019].

[7] J. Venegas Requena , «Codificadores Rotatorios,» 23 Mayo 2019. [En línea]. Available: http://ramos.elo.utfsm.cl/. [Último acceso: 2 Marzo 2019].

[8] A. O. LEÓN AGUIRRE y J. V. E TAPIA VACA, «"SINCRONISMO Y SUPERVISIÓN DE POSICIÓN Y VELOCIDAD DE UN MOTOR TRIFÁSICO ASÍNCRONO CON UN SISTEMA DE ENCODERS MAESTRO-ESCLAVO PARA APLICACIONES DE CONTROL DE EJES.”,» 19 Octubre 2019. [En línea]. Available: 
http://repositorio.espe.edu.ec/handle/21000/4237. [Último acceso: 12 Febrero 2019].

[9] M. MANCHENO, «MANUAL DE PRODUCCIÓN MÁS LIMPIA PARA LA ELABORACIÓN DE ESPONJA EN LA EMPRESA CICLA CIA LTDA,» 23 Junio 2010. [En línea]. Available: http://dspace.ucuenca.edu.ec/bitstream. [Último acceso: 21 Febrero 2019].

[10] G. T. Macanica, «Materiales para herramientas de corte,» 10 junio 2013. [En línea]. Available: http://www3.fi.mdp.edu.mz. [Último acceso: 2 Marzo 2019].

[11] D. E. Ososrio Orellana , «Estudio, Puesta en marcha y proposición de ensayos para un transportador de tornillo de laboratorio,» 22 Mayo 2018. [En línea]. Available: http://opac.pucv.cl/pucv_txt/txt4500/UCC4538_01. [Último acceso: 21 Febrero 2019].

[12] J. J. Zulaika, J. Lekunberri y J. Gorrotxa, «Concepción de accionamientos de alta velocidad basados en husillos a bolas,» 15 Mayo 1999. [En línea]. Available: https://www.schaeffler.com. [Último acceso: 12 Febrero 2019].

[13] M. Marrón Romera, «SENSORES PARA POSICIONAMIENTO DE ROBOTS MÓVILES,»Sensores de posicion, vol. 10, nº 7, p. 8, Junio 2014.

[14] A. . O. LEÓN AGUIRRE , «SINCRONISMO Y SUPERVISIÓN DE POSICIÓN Y VELOCIDAD DE UN MOTOR TRIFÁSICO ASÍNCRONO CON UN SISTEMA DE ENCODERS MAESTROESCLAVO PARA APLICACIONES DE CONTROL DE EJES,» 15 Octubre 2009. [En línea]. [Último acceso: 16 Marzo 2019].

[15] . E. Pilaquinga y A. Enríquez, «Implementacion de un sistema manual automatico para la maquina de cortes de esponja en la empresa Improespuma,»16 Junio 2010. [En línea]. Available: https://repositorio.espe.edu.ec/handle/21000/1651. [Último acceso: 15 Febrero 2019].

[16] Rubi, «Cortadoras de esponja manual a temperatura,» Corte de esponja, vol. 3, nº 4, p. 256, Enero 2018.

[17] C. I. Electrotécnica, «Lenguajes de programación-IEC 1131-3,» 27 Enero 2011. [En línea]. Available: https://www.iec.ch/. [Último acceso: 28 Febrero 2019].

[18] J. Fortes Monteiro, «Diseño e implementacion de encoders absolutos CANopen,» 13 Julio 2018. [En línea]. Available: https://earchivo.uc3m.es/handle/10016/6974. [Último acceso: 12 Febrero 2019].

[19] S. I.d. A. proporcionada, «Interfaces hombre-máquina para sistemas de automatización de procesos,» 9 Julio 2015. [En línea]. Available: http://wilmingtonisa.org. [Último acceso: 28 Febrero 2019].

[20] J. D. Rairan Antolines y J. M. Fonseca, «Algoritmo para la aproximación de la velocidad de giro de un eje mediante un encoder incremental,» 16 Junio 2017. [En línea]. Available: https://revistas.ja veriana.edu.co/index.php/iyu/article/view/1820. [Último acceso: 15 Febrero 2019].

[21] J. P. Celis Vargas, «Sistemas de control de posicion y velocidad para maquinas de CNC usando PLC,» 18 Septiembre 2010. [En línea]. Available: https://repository.upb.edu.co/handle/20.500.11912/1223. [Último acceso: 12 Febrero 2019].

[22] F. Acuña y H. Terán, «Diseño y construcción de un prototipo de cortadora por láser de dióxido de carbono con control numérico computarizado,» 15 Enero 2018. [En línea]. Available: http://repositorio.espe.edu.ec/handle/21000/9327. [Último acceso: 17 Febrero 2019].

[23] F. FONSECA PAREDES y M. . P. SANTO GUANOLUISA , «DISEÑO, CONSTRUCCIÓN E IMPLEMENTACIÓN DEL TORNILLO DE ARQUÍMEDES EN EL TUBO DE ESCAPE DEL VEHÍCULO CORSA WIND DEL LABORATORIO DIESEL GASOLINA, CON EL FIN DE ELEVAR LOS PARÁMETROS DE POTENCIA Y EFICIENCIA,» 18 Junio 2011. [En línea]. Available: http://repositorio.espe.edu.ec/handle/21000/4173. [Último acceso: 21 Junio 2019].

[24] Alibaba, «Cortadora De Espuma Poliuretano Alibaba,» Cortadoras de espuma, vol. $8, \mathrm{n}^{\circ} 3$, p. 8, Octubre 2016.
[25] L. M. Vega Romero, «Diseño e Implementación de un Sistema de Control de Velocidad y Posición Mediante un Servomotor para el Laboratorio de Accionamientos Eléctricos de la Universidad de las Fuerzas Armadas “ESPE” Extensión Latacunga,» 2016. [En línea].

[26] Baumer, «Machine engineering,» Maquinaria de corte, vol. 5, $\mathrm{n}^{\circ} 2$, p. 55, Enero 2018.

[27] . A. Angulo Romero y S. Franco Sánchez, «Sistema laser en el proceso de corte y marcado de materiales usando arduino uno,» 20 Septiembre 2017. [En línea]. Available: http://www.uppvirtual.org/revistas/index.php/RICCVA/article/view /103/92. [Último acceso: 15 Febrero 2019].

[28] N. J. Fuentes Alarcon y J. E. Hodge, «Fabricacion y automatizacion de una maquina para cortar cajas de carton con PLC,» 22 Mayo 2010 [En línea]. Available: https://repository.upb.edu.co/handle/20.500.11912/829. [Último acceso: 13 Febrero 13].

[29] S. d. t. y. p. social, «Sistemas de protección y dispositivos de seguridad en la maquinaria y equipo que se utilice en los centros de trabajo.,» 1 Mayo 1999. [En línea]. Available: http://www.dof.gob.mx/nota_detalle.php?codigo $=4948965 \&$ fecha $=$ 31/05/1999. [Último acceso: 28 Febrero 2019].

[30] D. F. Perez Esparza , «Estudio de factibilidad para la produccion y comercializacion de espumas flexibles de poliuretano en la ciudad de ambato,» 23 Junio 2015. [En línea]. Available: http://dspace.espoch.edu.ec/handle/123456789/4181. [Último acceso: 15 Febrero 2019]. 